\title{
TOP 30 SOFT SKILLS IN TOURISM AND HOSPITALITY GRADUATES: A SYSTEMATIC LITERATURE REVIEW
}

\author{
Ljudevit Pranić \\ Smiljana Pivčević \\ Daniela Garbin Praničević
}

https://doi.org//10.20867/tosee.06.43

\begin{abstract}
Purpose - To improve the employability of tourism and hospitality (T\&H) graduates, faculty must continually reassess what soft skills are essential for $\mathrm{T} \& \mathrm{H}$ graduates to possess and amend their curricula to meet these needs. Thus, this study aims to identify the soft skills perceived as essential for the T\&H graduates through a systematic literature review (SLR).

Methodology - Google Scholar was systematically queried from 1990 to April 2018, resulting in 77 T\&H soft skills-related peer-reviewed publications. Four T\&H researchers/educators produced an initial pool of $1447 \mathrm{~T} \& \mathrm{H}$ soft skills from these publications, categorized them in either conceptual/creative, leadership or interpersonal dimensions of Sandwith's (1993) Competency Domain Model and trimmed for relevance. Finally, the elicited key soft skills were further edited via an online survey of $19 \mathrm{~T} \& \mathrm{H}$ researchers/educators from Italy, Malta, Portugal, and Spain. The relevance of generated soft skills was double-verified thought same SLR based process for literature from April 2018 onwards.

Findings - A final set of 30 items emerged - 10 items per each of the three soft skills dimensions - i.e. conceptual/creative, leadership, and interpersonal. Based on these findings, both T\&H higher education institutions and faculty should evaluate their classes/curricula to determine whether a particular soft skill identified in this study is included as a learning objective of one or more specific classes.

Contribution - The main contribution lies in devising a theoretically-and methodologically-based list of key soft skills for a broad range of segments of the T\&H industry. From a methodological point of view, the SLR conducted is considered as novelty in T\&H soft skills research. Practically, this study provides educators with an updated benchmark tool to identify gaps in the curriculum. The related positive implications are expected for the students and T\&H industry as well.

Keywords: Soft skills, systematic literature review, key skills list, student employability, hospitality human resources, tourism education.
\end{abstract}

\section{INTRODUCTION}

Travel \& Tourism industry accounts for $10.4 \%$ of world GDP, supports $10 \%$ of jobs worldwide (319 million jobs), being the fourth biggest employment sector (WTTC 2019). In the EU, tourism is the third largest socio-economic activity and in 2019 it directly and indirectly supported over 37.1 million jobs, contributed to $9.1 \%$ of the total EU GDP (WTTC 2020). 
ToSEE - Tourism in Southern and Eastern Europe, Vol. 6, pp. 637-656, 2021.

Lj. Pranić, S. Pivčević, D. Garbin Praničević: TOP 30 SOFT SKILLS IN TOURISM AND

The worldwide growth and complexity of tourism has led to a demand for highly employable graduates and, in turn, to a dramatic increase in the number of Higher Education Institutions (HEI) offering baccalaureate programs in tourism and hospitality (Sisson and Adams 2013). Furthermore, HEIs spawn graduates with degrees not only in the traditional areas of lodging, food and beverage, and tourism but also in meetings, events, conventions, festivals, recreation, gaming, cruise management, etc. (Sisson and Adams 2013). Graduate employability can be defined as "a set of achievements - skills, understandings and personal attributes - that makes graduates more likely to gain employment and be successful in their chosen occupations, which benefits themselves, the workforce, the community and the economy" (Yorke 2006, 8).

Despite many studies on the topic, no consensus exists on which competencies are most important for the T\&H industry (Ferreras-Garcia et al. 2020). However, in the advent of fourth industrial revolution, the employability skills required from graduates are shifting from technically focused towards social and softer skills (Teng et al. 2019). Soft skills are essential in today's increasingly volatile labor market and necessary to increase graduate employability (Succi and Canovi 2020). In order to successfully transition from school to work, contemporary students need to develop a range of soft skills, such as entrepreneurial skills, coping skills, learning to learn, ability to work in teams, clear and effective communication, capacity to adapt to different cultural contexts, problem solving, conflict management, capability to endure complex or stressful situations, etc. (Succi 2019). The findings in T\&H industry confirm the prevailing importance of soft skills (Denizci Guillet et al. 2019).

In the quest to improve the employability of tourism graduates in the EU's rapidly evolving T\&H sector, HEIs' educators must continually investigate which soft skills are essential for $\mathrm{T} \& \mathrm{H}$ graduates to possess and amend the $\mathrm{T} \& \mathrm{H}$ curriculum to meet these needs (Sisson and Adams 2013). The main objective of this study is to identify the top 30 soft skills required from the $\mathrm{T} \& \mathrm{H}$ graduates. In order to encompass the voluminous literature and soft skills identified in the $\mathrm{T} \& \mathrm{H}$ literature, we apply the systematic literature review (SLR) classifying the soft skills based on Sandwith's (1993) Competency Domain Model (CDM).

\section{SOFT SKILLS AND THE COMPETENCY DOMAIN MODEL}

In both scholarly and professional literature, the set of work-related personal attributes, knowledge, and skills is often termed as competencies (Wang and Tsai 2014) or job competencies (Sisson and Adams 2013). These competencies can be further defined as hard or soft, depending on whether they cover vocational/technical aspects or interpersonal skills (Sisson and Adams 2013). However, what competencies HEIs offer may differ substantially from the actual competences that businesses need (Fraser 2020).

Traditionally, the curricula of HEIs in tourism has focused on learning hard skills (Wilks and Hemsworth 2011), sometimes termed technical skills, internal employability skills, vocational skills, specific skills, professional abilities, subject skills, specific techniques or employability capital (Wang and Tsai 2014; Weber et al. 2013). Because hard skills are considered task-oriented (i.e. administrative and technical) competencies learned 
ToSEE - Tourism in Southern and Eastern Europe, Vol. 6, pp. 637-656, 2021.

Lj. Pranić, S. Pivčević, D. Garbin Praničević: TOP 30 SOFT SKILLS IN TOURISM AND ..

through formal education and/or training (Weber et al. 2009), working in tourism has often been inaptly characterized as low skilled (Baum 2006).

Contrary to the traditional view, the contemporary educational paradigm increasingly places greater emphasis on soft skills (Shum et al. 2018), sometimes termed nontechnical skills, employability skills, external employability skills, core skills, people-related skills, personal skills, applied skills, 21st-century skills, generic skills, common skills, key skills, life skills, transversal skills or transferable skills (Robinson and Garton 2008; Robles 2012). Soft skills can be defined as the conceptual/creative, leadership, and interpersonal competencies that are necessary to apply hard skills in any position or field of work (Cimatti 2016; Weber et al. 2009).

Although both hard and soft skills are necessary for a successful T\&H career (Andrades and Dimanche 2017), soft skills are most often regarded as more important (Denizci Guillet et al. 2019), especially since they "can be deemed relevant across many different jobs or professions" (Cassidy 2006, 508) and their growing relevance in the foreseeable future (Teng et al. 2019). Thus, identifying the key soft skills that enhance T\&H employability is a valuable theoretical and practical goal.

A review of T\&H scholarly literature reveals that Sandwith's (1993) CDM (Figure 1) is often used as a descriptive tool to identify, categorize and summarize relevant competencies for a specific job (Chung-Herrera et al. 2003; Tsai et al. 2006). It divides competencies into (1) conceptual/creative, (2) leadership, (3) interpersonal, (4) administrative, and (5) technical domains.

\section{Figure 1: Sandwith's Competency Domain Model}

$$
\text { Hard skills } \quad \text { Soft skills }
$$

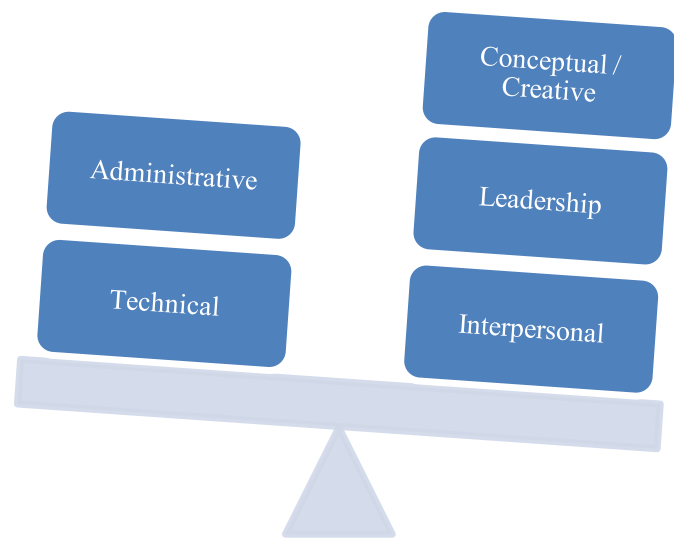

Source: Authors' elaboration based on Sandwith (1993) 
ToSEE - Tourism in Southern and Eastern Europe, Vol. 6, pp. 637-656, 2021.

Lj. Pranić, S. Pivčević, D. Garbin Praničević: TOP 30 SOFT SKILLS IN TOURISM AND ...

The conceptual/creative domain denotes "cognitive skills associated with comprehending important elements of the job" (Sandwith 1993, 46). The leadership domain encompasses the skills to communicate and execute an organization's current and new ideas and the ability to 'get everyone on board'. The interpersonal domain relates to how well an individual interacts with both work colleagues and customers. This domain includes oral, written, and telephone communication skills, as well as conflict management and negotiation skills (Sandwith 1993).

The administrative skills are typically the same across all departments of an organization and entail knowing about the rules and regulations that an organization must follow, educating others about them, and enforcing them. The technical domain comprises those skills that are essential to producing the product or service (Figure 1).

In terms of the hard vs. soft skills dichotomy, while the technical and administrative skills correspond to hard competencies, the skills in the conceptual, leadership and interpersonal domains correspond to soft skills (Weber et al. 2009). CDM is one of the most influential and reliable competency models (Millar et al. 2010) and the prevailing model used in hospitality research (Marneros 2020), and thus adopted in this study to classify soft skills in $\mathrm{T} \& \mathrm{H}$.

\section{METHODOLOGY}

In line with Khan et al. (2003), this study followed a five-step SLR, i.e. (1) framing the question for a review, (2) identifying relevant work, (3) assessing the quality of studies, (4) summarizing the evidence, and (5) interpreting the findings (Figure 2). 
ToSEE - Tourism in Southern and Eastern Europe, Vol. 6, pp. 637-656, 2021.

Lj. Pranić, S. Pivčević, D. Garbin Praničević: TOP 30 SOFT SKILLS IN TOURISM AND ...

\section{Figure 2: A Five-Step Systematic Literature Review (SLR)}

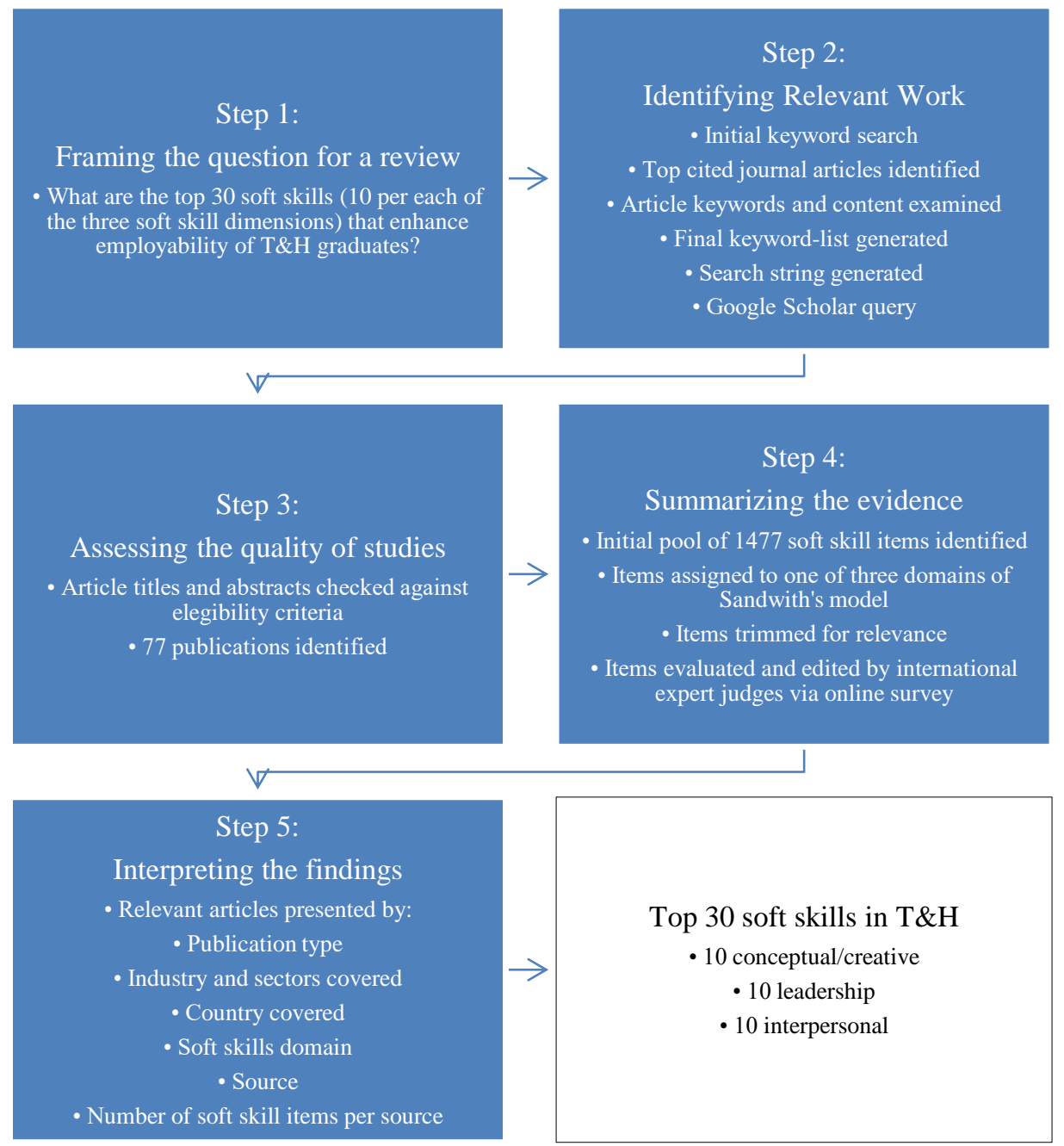

\subsection{Framing the Question for a Review}

Our review was designed to answer the following question: What are the top 10 soughtafter soft skills that enhance employability of T\&H graduates in each of the three soft skills dimensions?

\subsection{Identifying Relevant Work}

Google Scholar, the most comprehensive data source across almost all subject areas (Gusenbauer 2019; Martin-Martin et al. 2021, 2018a, 2018b), was systematically queried 
ToSEE - Tourism in Southern and Eastern Europe, Vol. 6, pp. 637-656, 2021.

Lj. Pranić, S. Pivčević, D. Garbin Praničević: TOP 30 SOFT SKILLS IN TOURISM AND ..

and mined to identify scholarly works related to skills /competencies in T\&H. The search included works from 1990 to April of 2018.

The first phase consisted of defining relevant keywords to be used to search for scholarly sources on T\&H soft skills. Initiating the query with the search string 'skills AND (tourism OR hospitality)' produced a number of search results. Thus, top 20 cited articles on T\&H skills were selected and their keywords and article content examined. The resulting keyword-list comprised the following terms: soft skills, employability skills, nontechnical skills, core skills, people-related skills, personal skills, applied skills, twenty-first century skills, generic skills, common skills, key skills, transferable skills, and life skills.

The second phase consisted of searching Google Scholar using a search string broadened with the terms from the previously generated keyword-list, i.e. '(soft OR employability OR nontechnical OR core OR people-related OR personal OR applied OR twenty-first century OR generic OR common OR key OR transferable OR life) AND (skills OR competencies) AND (tourism OR hospitality). Since Google Scholar's default search setting is to use the search string to search the full text of its scholarly works, including citations and abstracts, we did a second search using Google Scholar's advanced search feature "intitle:" in order to find results with our search term in the document title only. Ultimately, step two generated a large number of publications for further assessment.

\subsection{Assessing the quality of studies}

In step three, titles and abstracts of retrieved publications were checked against eligibility requirements. We sought peer-reviewed publications (i.e., journal articles, review pieces, conceptual pieces, and conference proceedings) published in English that provided information on soft skills in $\mathrm{T} \& \mathrm{H}$.

Four T\&H researchers/educators from a large public university in Croatia divided the publications in a way that each title and abstract was evaluated by two reviewers. An assessment form was posted to a shared collaboration site, and each reviewer independently assessed whether a publication met the inclusion criteria. Discrepancies were resolved by the two reviewers jointly reviewing a study in question. If no consensus was reached, a third reviewer, unaware of the other reviewers' decisions, functioned as an arbitrator. After thorough selection, step three resulted in 77 scholarly works that were germane to our review question. Each above presented phases was carried out in the same way for works from both sets as defined above.

\subsection{Summarizing the evidence}

In the fourth step, applying the same evaluation procedure as in step three (i.e., independent assessment, joint resolution of discrepancies, and arbitration), the four reviewers thoroughly read the selected publications and extracted the soft skills. 
ToSEE - Tourism in Southern and Eastern Europe, Vol. 6, pp. 637-656, 2021.

Lj. Pranić, S. Pivčević, D. Garbin Praničević: TOP 30 SOFT SKILLS IN TOURISM AND

The first phase consisted of produced an initial pool of all T\&H soft skills elicited from SLR identified publications. The second phase entailed placing the soft skills from the initial item pool in either conceptual/creative, leadership or interpersonal dimensions of Sandwith's (1993) CDM. When a reviewer was in doubt of competency domain, that skill was temporarily placed in the unclassified category and later jointly discussed and allocated to one of the three domains. In phase three, through an iterative consultation process, the reviewers checked and ranked the items for relevance, clarity, completeness, and redundancy and to produce a smaller, more manageable item pool for further consideration (DeVellis 2003, 1991).

In phase four, in mid 2018, the soft skills were further evaluated and edited via an online survey of 19 T\&H researchers/educators from Italy, Malta, Portugal, and Spain. The participants were instructed to perform one or more of the following four actions: (1) mark the items on the trimmed-down list that should be deleted, (2) reword the items on the trimmed-down list, (3) mark the items in the initial item pool that should be added to the trimmed-down list of soft skills, and/or (4) leave it as it is. Systematizing the responses received, the final list of top $30 \mathrm{~T} \& \mathrm{H}$ soft skills was obtained.

In all four phases of summarizing the evidence, participants were asked to ensure that a set of desirable soft skills that an entry-level management trainee should possess upon completion of a $\mathrm{T} \& \mathrm{H}$ undergraduate degree is applicable to any segment of the $\mathrm{T} \& \mathrm{H}$ industry. All four phases also helped ascertain face, content, and construct validity.

As the research process described was conducted in 2018, to ascertain that its results provide original contribution to the literature and that soft skills extracted are relevant, the steps $1-3$ and first two phases of step 4 of SLR (Figure 2) were performed for the period from April 2018 to April 2021. This yielded 22 publications that were analyzed as previous studies in presenting the findings, where, relevant these two periods are separately reported.

\subsection{Interpreting the findings}

The literature matrix catalogued the relevant articles by publication type (industries and sectors covered, countries covered, soft skills domain, source (author and year of publication), and number of soft skill items per source. Systematized features of papers analysed (Table 1) reveal that scholars have looked into the soft skills in tourism from diverse perspectives but empirical studies are prevailing. Several empirical studies use qualitative or mixed method approach whilst the majority are survey-based focusing on separate individual stakeholders in the T\&H HEI process or more of them (so-called "gap studies"). The studies are conducted worldwide, the majority being in the USA. 
ToSEE - Tourism in Southern and Eastern Europe, Vol. 6, pp. 637-656, 2021.

Lj. Pranić, S. Pivčević, D. Garbin Praničević: TOP 30 SOFT SKILLS IN TOURISM AND ...

\section{Table 1: The typology of scholarly works covered}

\begin{tabular}{|c|c|c|}
\hline \multirow{2}{*}{\multicolumn{2}{|c|}{ Conceptual pieces }} & Authors \\
\hline & & $\begin{array}{l}\text { Sandwith (1993); Burns (1997); Bharwani \& Jauhari (2013); Caruana \& } \\
\text { Mcpherson (2014); Bharwani \& Talib (2017); Ginaya et al (2020); Kumar (2021) }\end{array}$ \\
\hline Revi & ew pieces & Klein et al. (2006); Adeyinka-Ojo (2018) \\
\hline \multirow{5}{*}{ : } & $\begin{array}{l}\text { Surveying } \\
\text { students }\end{array}$ & $\begin{array}{l}\text { Hannam et al. (2004); Horng \& Lu (2006); Hertzman et al. (2015); Ko (2010); } \\
\text { Spowart (2011); Scarinci \& Pearce (2011); Valdez et al. (2015); Christou \& } \\
\text { Chatzigeorgiou (2019); Shereni (2019); Patiar et al. (2020); Susaeta et al. (2020); } \\
\text { Chen et al. (2021) }\end{array}$ \\
\hline & $\begin{array}{l}\text { Surveying } \mathrm{T} \& \mathrm{H} \\
\text { industry } \\
\text { professionals }\end{array}$ & $\begin{array}{l}\text { Baum (1990); Clark (1993); Brownell (1994); Tas et al. (1996); Shay \& Tracey } \\
\text { (1997); Siu (1998); Kay \& Russette (2000); Kriegl (2000); Lin (2002);Chung- } \\
\text { Herrera et al. (2003); Tesone \& Ricci (2006); Baum (2007); Baum and Thomson } \\
\text { (2007); Brownell (2008); Zehrer \& Mössenlechner (2009); Hassan et al. (2009); } \\
\text { Foster et al. (2010); Kalargyrou \& Woods (2011); Causin \& Ayoun (2011); Wilks } \\
\text { \& Hemsworth (2011); Dhiman (2012); Weber et al. (2013); Sisson \& Adams } \\
\text { (2013); Wang (2013); Lolli (2013); Yang et al. (2015); Yang et al. (2016); Chiu et } \\
\text { al. (2016); Shum et al. (2018); Bello et al. (2019); Costa et al. (2019); Andrades \& } \\
\text { Dimanche (2019);Denizci Guillet et al. (2019); Ferreras-Garcia et al.(2019); } \\
\text { Kimeto (2020); Marneros et al. (2020) }\end{array}$ \\
\hline & $\begin{array}{l}\text { Surveying } \\
\text { university } \\
\text { professors }\end{array}$ & Fraser (2020); Hossny (2020) \\
\hline & $\begin{array}{l}\text { Surveying } \\
\text { multiple } \\
\text { stakeholders }\end{array}$ & $\begin{array}{l}\text { Bach \& Milman (1996); Davies (2000); Tsai et al. (2006); Wilkins (2006); Munar } \\
\text { \& Montaño (2009); Cheung et al. (2010); Millar et al. (2010); Huang \& Lin (2010); } \\
\text { Suh et al. (2012); Yang et al. (2014); Moolman \& Wilkinson (2014); Luka (2015); } \\
\text { Huang et al. (2016); Jack et al. (2017); Jiang \& Alexakis (2017); Kim et al. (2017); } \\
\text { Vučetić (2018); Raybould \& Anderson \& Sanga (2018); Bustreo et al.(2018); } \\
\text { Sharma \& Sharma (2019); Deale \& Lee (2019); Temizkan (2020); Marneros et al. } \\
\text { (2021) }\end{array}$ \\
\hline & $\begin{array}{l}\text { Surveying } \\
\text { professionals in } \\
\text { various } \\
\text { industries }\end{array}$ & Wesley et al. (2017) \\
\hline \multirow{4}{*}{ 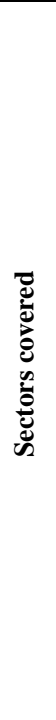 } & Hotels & $\begin{array}{l}\text { Brownell (1994); Tas et al. (1996); Shay \& Tracey (1997); Siu (1998); Kay \& } \\
\text { Russette (2000); Lin (2002); Chung-Herrera et al. (2003); Tsai et al. (2006); } \\
\text { Raybould \& Wilkins (2006); Baum (2007); Huang \& Lin (2010); Cheung et al. } \\
\text { (2010); Kalargyrou \& Woods (2011); Wilks \& Hemsworth (2011); Wang \& Tsai } \\
\text { (2014); Yang et al. (2015); Yang et al. (2016); Fraser (2020); Hossny (2020); } \\
\text { Marneros et al. (2020); Temİkan (2020); Patiar et al. (2020); Marneros et al. (2021) }\end{array}$ \\
\hline & $\begin{array}{l}\text { Food \& } \\
\text { Beverage }\end{array}$ & Wang (2013); Wang \& Tsai (2014); Sharma \& Sharma (2019); Shereni (2019) \\
\hline & $\begin{array}{l}\text { Other T\&H } \\
\text { businesses }\end{array}$ & $\begin{array}{l}\text { Clark (1993); Davies (2000); Kriegl (2000); Nickson et al. (2005); Horng \& Lu } \\
\text { (2006); Tesone \& Ricci (2006); Baum and Thomson (2007); Brownell (2008); } \\
\text { Zehrer \& Mössenlechner (2009); Hassan et al. (2009); Foster et al. (2010); Millar } \\
\text { et al. (2010); Scarinci \& Pearce (2011); Causin \& Ayoun (2011); Dhiman (2012); } \\
\text { Sisson \& Adams (2013); Lolli (2013); Moolman \& Wilkinson (2014); Hertzman et } \\
\text { al. (2015); Valdez et al. (2015); Luka (2015); Kim et al. (2017); Wesley et al. } \\
\text { (2017); Jack et al. (2017); Jiang \& Alexakis (2017); Vučetić (2018); Bustreo et } \\
\text { al.(2018); Shum et al. (2018); Bello et al.(2019); Costa et al. (2019); Ferreras- } \\
\text { Garcia et al.(2019); Andrades \& Dimanche (2019); Deale \& Lee (2019); Kimeto } \\
\text { (2020), Susaeta et al. (2020); Chen et al. (2021); Kumar (2021) }\end{array}$ \\
\hline & Other & $\begin{array}{l}\text { Baum (1990); Sandwith (1993); Bach \& Milman(1996); Hannam et al. (2004); } \\
\text { Klein et al. (2006); Munar \& Montaño (2009); Ko (2010); Huang et al. (2016); Chiu } \\
\text { et al. (2016); Wesley et al. (2017); Anderson \& Sanga (2018), Denizci Guillet et al. } \\
\text { (2019); Christou \& Chatzigeorgiou (2019); Ginaya et al. (2020) }\end{array}$ \\
\hline
\end{tabular}


ToSEE - Tourism in Southern and Eastern Europe, Vol. 6, pp. 637-656, 2021.

Lj. Pranić, S. Pivčević, D. Garbin Praničević: TOP 30 SOFT SKILLS IN TOURISM AND ..

Table 1 (continued)

\begin{tabular}{|c|c|c|}
\hline & & Authors \\
\hline \multirow{29}{*}{ 递 } & Australia & Raybould \& Wilkins (2006); Patiar et al. (2020); Fraser (2020) \\
\hline & Austria & Zehrer \& Mössenlechner (2009) \\
\hline & China & Siu (1998); Cheung et al. (2010); Yang et al. (2015); Yang et al. (2016) \\
\hline & Cyprus & Marneros et al. (2020); Marneros et al. (2021) \\
\hline & Egypt & Hossny (2020) \\
\hline & Greece & Christou \& Chatzigeorgiou (2019) \\
\hline & Hong Kong & Denizci Guillet et al. (2019) \\
\hline & India & Dhiman (2012); Yang et al. (2014); Sharma \& Sharma (2019) \\
\hline & Indonesia & Ginaya et al (2020) \\
\hline & Italy & Bustreo et al. (2018) \\
\hline & Kenya & Kimeto (2020) \\
\hline & Korea & Kim et al. (2017) \\
\hline & Kyrgyzstan & Baum (2007); Baum and Thomson (2007) \\
\hline & Malawi & Bello et al. (2019) \\
\hline & Malaysia & Hassan et al. (2009); Chiu et al. (2016) \\
\hline & $\begin{array}{l}\text { Mongolia and } \\
\text { Uzbekistan }\end{array}$ & Baum and Thomson (2007) \\
\hline & Montenegro & Vučetić (2018) \\
\hline & Philippines & Valdez et al. (2015) \\
\hline & Portugal & Wilks \& Hemsworth (2011); Costa et al. (2019) \\
\hline & Russia & Andrades \& Dimanche (2019) \\
\hline & South Africa & Spowart (2011); Moolman \& Wilkinson (2014) \\
\hline & Spain & Munar \& Montaño (2009); Ferreras-Garcia et al. (2019); Susaeta et al. (2020) \\
\hline & Taiwan & $\begin{array}{l}\text { Lin (2002); Horng \& Lu (2006); Tsai et al. (2006); Ko (2010); Huang \& Lin (2010) } \\
\text { Wang (2013); Wang \& Tsai (2014); Chen et al. (2021) }\end{array}$ \\
\hline & Tanzania & Anderson \& Sanga (2018) \\
\hline & Turkey & Temzkan (2020) \\
\hline & UK & Clark (1993); Hannam et al. (2004); Nickson et al. (2005); Foster et al. (2010) \\
\hline & USA & $\begin{array}{l}\text { Baum (1990); Brownell (1994); Tas et al. (1996); Bach \& Milman(1996); Davies } \\
\text { (2000); Kay \& Russette (2000); Tesone \& Ricci (2006); Brownell (2008); Millar et } \\
\text { al. (2010);Causin \& Ayoun (2011); Kalargyrou \& Woods (2011); Scarinci \& } \\
\text { Pearce (2011); Suh et al. (2012); Lolli (2013); Weber et al. (2013); Sisson \& Adams } \\
\text { (2013); Hertzman et al. (2015); Huang et al. (2016); Jack et al. (2017);Jiang \& } \\
\text { Alexakis (2017); Wesley et al. (2017); Shum et al. (2018); Deale \& Lee (2019) }\end{array}$ \\
\hline & $\begin{array}{l}\text { Worldwide / } \\
\text { International }\end{array}$ & $\begin{array}{l}\text { Shay \& Tracey (1997); Kriegl (2000); Chung-Herrera et al. (2003); Klein et al. } \\
\text { (2006); Luka (2015); Kumar (2021) }\end{array}$ \\
\hline & Zimbabve & Shereni (2019) \\
\hline
\end{tabular}

Source: Author's research

The soft skills categorized according to the domain and the period of publishing are enclosed in Table 2. The number of soft skills revealed in 1990-2018 period (1180 skills) demonstrated the need for the present study to establish the most important soft skills in T\&H. The set of scholarly works published after March 2018 indicates that soft skills remain relevant and in the focus of interest of scientific community (Figure 3). More importantly, the detailed analysis of papers in that period revealed that no papers were found applying similar extensive methodological process for identifying the key soft skills in $\mathrm{T} \& \mathrm{H}$. 
ToSEE - Tourism in Southern and Eastern Europe, Vol. 6, pp. 637-656, 2021.

Lj. Pranić, S. Pivčević, D. Garbin Praničević: TOP 30 SOFT SKILLS IN TOURISM AND ...

Table 2: An Initial Pool of Soft Skill Items

\begin{tabular}{|c|c|c|c|c|c|}
\hline \multirow[b]{2}{*}{$\begin{array}{c}\text { Soft } \\
\text { skill } \\
\text { domain }\end{array}$} & \multicolumn{3}{|c|}{1990 - 2018 (March) } & \multicolumn{2}{|c|}{2018 (April) - 2021 (April) } \\
\hline & $\begin{array}{c}\text { Soft } \\
\text { skill } \\
\text { items } \\
(\mathrm{No})\end{array}$ & & ors & $\begin{array}{c}\text { Soft } \\
\text { skill } \\
\text { items } \\
(\mathrm{No})\end{array}$ & Authors \\
\hline 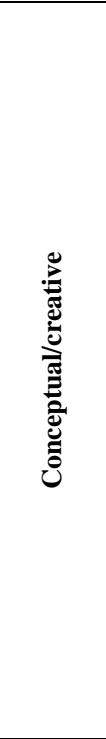 & 289 & $\begin{array}{l}\text { Bach \& Milman (1996) } \\
\text { Brownell (2008) } \\
\text { Causin \& Ayoun (2011) } \\
\text { Dhiman (2012) } \\
\text { Foster et al. (2010) } \\
\text { Hannam et al. (2004) } \\
\text { Hassan et al. } \\
\text { (2009) } \\
\text { Hertzman et al. (2015) } \\
\text { Horng \& Lu (2006) } \\
\text { Huang \& Lin (2010) } \\
\text { Huang et al. (2016) } \\
\text { Jack et al. (2017) } \\
\text { Jiang \& Alexakis (2017) } \\
\text { Kalargyrou \& Woods } \\
\text { (2011) } \\
\text { Kay \& Russette (2000) } \\
\text { Kim et al. (2017) } \\
\text { Ko (2010) } \\
\text { Kriegl (2000) } \\
\text { Millar et al. (2010) }\end{array}$ & $\begin{array}{l}\text { Moolman \& Wilkinson } \\
\text { (2014) } \\
\text { Munar \& Montaño } \\
\text { (2009) } \\
\text { Raybould \& Wilkins } \\
\text { (2006) } \\
\text { Sandwith (1993) } \\
\text { Scarinci \& Pearce (2011) } \\
\text { Shay \& Tracey (1997) } \\
\text { Spowart (2011) } \\
\text { Suh et al. (2012) } \\
\text { Tas et al. (1996) } \\
\text { Tesone \& Ricci (2006) } \\
\text { Tsai et al. (2006) } \\
\text { Wang (2013) } \\
\text { Wilks \& Hemsworth } \\
\text { (2011) } \\
\text { Yang et al. (2014) } \\
\text { Valdez et al. (2015) } \\
\text { Yang et al. (2015) } \\
\text { Yang et al. (2016) } \\
\text { Zehrer \& Mössenlechner } \\
\text { (2009) }\end{array}$ & 43 & $\begin{array}{l}\text { Andrades \& } \\
\text { Dimanche (2019) } \\
\text { Bello et al.(2019) } \\
\text { Ferreras-Garcia et al. } \\
\text { (2019) } \\
\text { Fraser (2020) } \\
\text { Kimeto (2020) } \\
\text { Kumar (2021) } \\
\text { Marneros et al. } \\
\text { (2020) } \\
\text { Marneros et al. } \\
\text { (2021) } \\
\text { Patiar et al. (2020) } \\
\text { Shum et al. (2018) } \\
\text { Temizkan (2020) } \\
\text { Vučetić (2018) }\end{array}$ \\
\hline 氖 & 408 & $\begin{array}{l}\text { Bach \& Milman (1996) } \\
\text { Baum (1990) } \\
\text { Baum (2007) } \\
\text { Baum and Thomson } \\
\text { (2007) } \\
\text { Brownell (2008) } \\
\text { Causin \& Ayoun (2011) } \\
\text { Cheung et al. (2010) } \\
\text { Chung-Herrera et al. } \\
\text { (2003) } \\
\text { Clark (1993) } \\
\text { Dhiman (2012) } \\
\text { Foster et al. (2010) } \\
\text { Hannam et al. (2004) } \\
\text { Hertzman et al. (2015) } \\
\text { Horng \& Lu (2006) } \\
\text { Huang \& Lin (2010) } \\
\text { Huang et al. (2016) } \\
\text { Jack et al. (2017) } \\
\text { Jiang \& Alexakis (2017) } \\
\text { Kalargyrou \& Woods } \\
\text { (2011) } \\
\text { Kay \& Russette (2000) } \\
\text { Kim et al. (2017) } \\
\text { Ko (2010) } \\
\text { Kriegl (2000) } \\
\text { Luka (2015) }\end{array}$ & $\begin{array}{l}\text { Millar et al. (2010) } \\
\text { Moolman \& Wilkinson } \\
\text { (2014) } \\
\text { Munar \& Montaño } \\
\text { (2009) } \\
\text { Raybould \& Wilkins } \\
\text { (2006) } \\
\text { Sandwith (1993) } \\
\text { Scarinci \& Pearce (2011) } \\
\text { Shay \& Tracey (1997) } \\
\text { Siu (1998) } \\
\text { Spowart (2011) } \\
\text { Tas et al. (1996) } \\
\text { Tesone \& Ricci (2006) } \\
\text { Tsai et al. (2006) } \\
\text { Valdez et al. (2015) } \\
\text { Wang \& Tsai (2014) } \\
\text { Wang (2013) } \\
\text { Weber et al. (2013) } \\
\text { Wesley et al. (2017) } \\
\text { Wilks \& Hemsworth } \\
\text { (2011) } \\
\text { Yang et al. (2014) } \\
\text { Yang et al. (2015) } \\
\text { Yang et al. (2016) } \\
\text { Zehrer \& Mössenlechner } \\
\text { (2009) }\end{array}$ & 108 & $\begin{array}{l}\text { Andrades \& } \\
\text { Dimanche (2019) } \\
\text { Bustreo et al.(2018) } \\
\text { Chen et al.(2021) } \\
\text { Christou \& } \\
\text { Chatzigeorgiou } \\
\text { (2019) } \\
\text { Deale \& Lee (2019) } \\
\text { Denizci Guillet et al. } \\
\text { (2019) } \\
\text { Ferreras-Garcia et al. } \\
\text { (2019) } \\
\text { Fraser, (2020) } \\
\text { Ginaya et al. (2020) } \\
\text { Hossny (2018) } \\
\text { Kimeto (2020) } \\
\text { Kumar (2021) } \\
\text { Marneros et al. } \\
\text { (2020) } \\
\text { Marneros et al. } \\
\text { (2021) } \\
\text { Susaeta et al. (2020) } \\
\text { Temİzkan (2020) } \\
\text { Vučetić (2018) }\end{array}$ \\
\hline
\end{tabular}


ToSEE - Tourism in Southern and Eastern Europe, Vol. 6, pp. 637-656, 2021.

Lj. Pranić, S. Pivčević, D. Garbin Praničević: TOP 30 SOFT SKILLS IN TOURISM AND ...

Table 2 (continued)

\begin{tabular}{|c|c|c|c|c|c|}
\hline \multirow[b]{2}{*}{$\begin{array}{c}\text { Soft } \\
\text { skill } \\
\text { domain }\end{array}$} & \multicolumn{3}{|c|}{1990 - 2018 (March) } & \multicolumn{2}{|c|}{2018 (April) - 2021 (April) } \\
\hline & $\begin{array}{c}\text { Soft } \\
\text { skill } \\
\text { items } \\
(\mathrm{No}) \\
\end{array}$ & & ors & $\begin{array}{c}\text { Soft } \\
\text { skill } \\
\text { items } \\
(\mathrm{No}) \\
\end{array}$ & Authors \\
\hline 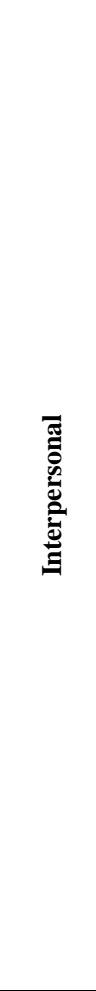 & 395 & $\begin{array}{l}\text { Bach \& Milman (1996) } \\
\text { Baum (1990) } \\
\text { Baum (2007) } \\
\text { Baum and Thomson } \\
\text { (2007) } \\
\text { Brownell (1994) } \\
\text { Brownell (2008) } \\
\text { Causin \& Ayoun (2011) } \\
\text { Cheung et al. (2010) } \\
\text { Chiu et al. (2016) } \\
\text { Clark (1993) } \\
\text { Davies (2000) } \\
\text { Dhiman (2012) } \\
\text { Foster et al. (2010) } \\
\text { Hannam et al. (2004) } \\
\text { Hassan et al. (2009) } \\
\text { Hertzman et al. (2015) } \\
\text { Horng \& Lu (2006) } \\
\text { Huang \& Lin (2010) } \\
\text { Huang et al. (2016) } \\
\text { Jack et al. (2017) } \\
\text { Jiang \& Alexakis (2017) } \\
\text { Kalargyrou \& Woods } \\
\text { (2011) } \\
\text { Kay \& Russette (2000) } \\
\text { Kim et al. (2017) } \\
\text { Klein et al. (2006) } \\
\text { Ko (2010) } \\
\text { Kriegl (2000) }\end{array}$ & $\begin{array}{l}\text { Lin (2002) } \\
\text { Lolli (2013) } \\
\text { Luka (2015) } \\
\text { Millar et al. (2010) } \\
\text { Moolman \& Wilkinson } \\
\text { (2014) } \\
\text { Munar \& Montaño } \\
\text { (2009) } \\
\text { Nickson et al. (2005) } \\
\text { Raybould \& Wilkins } \\
\text { (2006) } \\
\text { Robles (2012) } \\
\text { Sandwith (1993) } \\
\text { Scarinci \& Pearce (2012) } \\
\text { Shay \& Tracey (1997) } \\
\text { Siu (1998) } \\
\text { Spowart (2011) } \\
\text { Suh et al. (2012) } \\
\text { Tas et al. (1996) } \\
\text { Tesone \& Ricci (2006) } \\
\text { Tsai et al. (2006) } \\
\text { Valdez et al. (2015) } \\
\text { Weber et al. (2013) } \\
\text { Wesley et al. (2017) } \\
\text { Wilks \& Hemsworth } \\
\text { (2011) } \\
\text { Yang et al. (2014) } \\
\text { Yang et al. (2015) } \\
\text { Yang et al. (2016) } \\
\text { Zehrer \& Mössenlechner } \\
\text { (2009) }\end{array}$ & 95 & $\begin{array}{l}\text { Andrades \& } \\
\text { Dimanche (2019) } \\
\text { Christou \& } \\
\text { Chatzigeorgiou } \\
\text { (2019) } \\
\text { Deale \& Lee (2019) } \\
\text { Denizci Guillet et al. } \\
\text { (2019) } \\
\text { Ferreras-Garcia et al. } \\
\text { (2019) } \\
\text { Fraser (2020) } \\
\text { Ginaya et al. (2020) } \\
\text { Hossny (2018) } \\
\text { Kimeto (2020) } \\
\text { Kumar (2021) } \\
\text { Marneros et al. } \\
\text { (2020) } \\
\text { Marneros et al. } \\
\text { (2021) } \\
\text { Patiar et al. (2020) } \\
\text { Susaeta et al. (2020) } \\
\text { Temİzkan (2020) } \\
\text { Vučetić (2018) }\end{array}$ \\
\hline 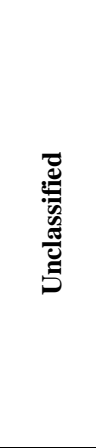 & 88 & $\begin{array}{l}\text { Hassan et al. (2009) } \\
\text { Hertzman et al. (2015) } \\
\text { Huang \& Lin (2010) } \\
\text { Huang et al. (2016) } \\
\text { Horng \& Lu (2006) } \\
\text { Jiang \& Alexakis (2017) } \\
\text { Kalargyrou \& Woods } \\
(2011) \\
\text { Ko (2010) }\end{array}$ & $\begin{array}{l}\text { Moolman \& Wilkinson } \\
\text { (2014) } \\
\text { Munar \& Montaño } \\
\text { (2009) } \\
\text { Scarinci \& Pearce (2011) } \\
\text { Sisson \& Adams (2013) } \\
\text { Wang (2013) } \\
\text { Wesley et al. (2017) } \\
\text { Yang et al. (2014) } \\
\text { Yang et al. (2015) } \\
\text { Yang et al. (2016) } \\
\text { Zehrer \& Mössenlechner } \\
\text { (2009) } \\
\text { Zhang (2012) }\end{array}$ & 51 & $\begin{array}{l}\text { Anderson (2018) } \\
\text { Chen et al.(2021) } \\
\text { Costa et al. (2019) } \\
\text { Ferreras-Garcia et al. } \\
\text { (2019) } \\
\text { Ginaya et al. (2020) } \\
\text { Sharma \& Sharma } \\
\text { (2019) } \\
\text { Shereni (2019) } \\
\text { Susaeta et al. (2020) }\end{array}$ \\
\hline $\begin{array}{l}\text { Total } \\
(1477)\end{array}$ & 1180 & & & 297 & \\
\hline
\end{tabular}

Source: Author's reseach 
ToSEE - Tourism in Southern and Eastern Europe, Vol. 6, pp. 637-656, 2021.

Lj. Pranić, S. Pivčević, D. Garbin Praničević: TOP 30 SOFT SKILLS IN TOURISM AND ..

The scholarly works published after March 2018 confirm the growing trend in publications dealing with the soft skills in $\mathrm{T} \& \mathrm{H}$, demonstrating the highest number of publications studying soft skills in T\&H (Figure 3).

\section{Figure 3: Trendline of publications dealing with skills in $\mathbf{T} \& H$}

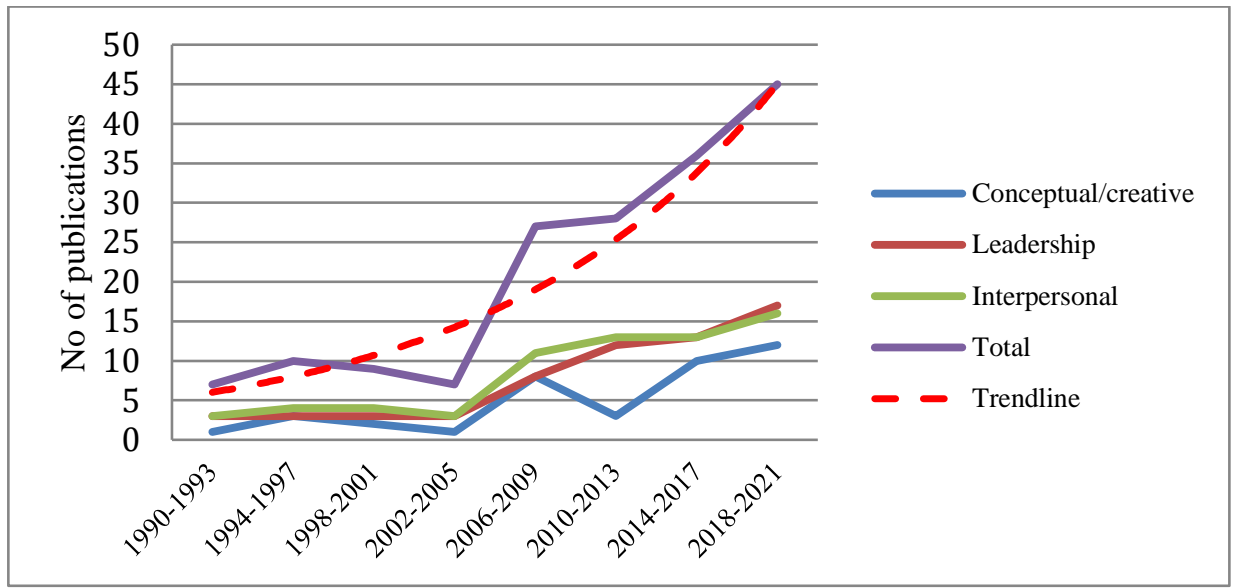

Source: Author research

Finally, Table 3 presents the top 30 soft skills in three soft skills domains based on the SLR for period 1990 and March 2018 and confirmed by 19 T\&H researchers/educators from 5 countries. The relevant skills were double-checked and found to be covered i.e still relevant in works published after March 2018.

\section{Table 3: The set of most desirable soft skills for $\mathbf{T} \& \mathbf{H}$ graduates}

\begin{tabular}{lll}
\hline \multicolumn{1}{c}{ Conceptual/Creative } & \multicolumn{1}{c}{ Leadership } & \multicolumn{1}{c}{ Interpersonal } \\
\hline $\begin{array}{l}\text { Maintaining the "big } \\
\text { picture" while working on a } \\
\text { specific property- } \\
\text { management, layout, and } \\
\text { design component }\end{array}$ & $\begin{array}{l}\text { Steering conflicts away from } \\
\text { personalities and toward } \\
\text { issues }\end{array}$ & $\begin{array}{l}\text { Managing guest requests } \\
\text { with understanding and } \\
\text { sensitivity }\end{array}$ \\
\hline $\begin{array}{l}\text { Manifesting critical thinking } \\
\text { Presenting ideas in a } \\
\text { convincing manner }\end{array}$ & $\begin{array}{l}\text { Communicating efficiently } \\
\text { (orally and in writing) with } \\
\text { other employees, clients, and } \\
\text { customers }\end{array}$ \\
$\begin{array}{l}\text { Demonstrating an } \\
\text { understanding of the } \\
\text { organization's interaction } \\
\begin{array}{l}\text { with the larger external } \\
\text { environment }\end{array}\end{array}$ & $\begin{array}{l}\text { Expressing disagreement in } \\
\text { a tactful and sensitive } \\
\text { manner }\end{array}$ & $\begin{array}{l}\text { Exhibiting emotional } \\
\text { intelligence, such as } \\
\text { emotional control and } \\
\text { passion }\end{array}$ \\
\hline
\end{tabular}


ToSEE - Tourism in Southern and Eastern Europe, Vol. 6, pp. 637-656, 2021.

Lj. Pranić, S. Pivčević, D. Garbin Praničević: TOP 30 SOFT SKILLS IN TOURISM AND ...

Table 3 (continued)

\begin{tabular}{|c|c|c|}
\hline Conceptual/Creative & Leadership & Interpersonal \\
\hline Developing new ideas & $\begin{array}{l}\text { Working to understand why } \\
\text { others resist change instead } \\
\text { of forcing others to accept } \\
\text { change }\end{array}$ & $\begin{array}{l}\text { Effectively handling staff } \\
\text { grievances and complaints }\end{array}$ \\
\hline $\begin{array}{l}\text { Developing adaptation } \\
\text { responses to unexpected } \\
\text { changes }\end{array}$ & $\begin{array}{l}\text { Operating effectively and } \\
\text { calmly under pressure or in } \\
\text { crisis situations }\end{array}$ & $\begin{array}{l}\text { Acting courteously and } \\
\text { respectfully }\end{array}$ \\
\hline $\begin{array}{l}\text { Exhibiting knowledge of } \\
\text { related job roles - peers, } \\
\text { subordinates, others }\end{array}$ & $\begin{array}{l}\text { Building networks with } \\
\text { people inside and outside the } \\
\text { firm }\end{array}$ & $\begin{array}{l}\text { Demonstrating awareness of } \\
\text { others' feelings }\end{array}$ \\
\hline $\begin{array}{l}\text { Manifesting development } \\
\text { and adaptation of innovation } \\
\text { or improvements }\end{array}$ & $\begin{array}{l}\text { Confronting problems early } \\
\text { before they become } \\
\text { unmanageable }\end{array}$ & Exhibiting negotiating skills \\
\hline $\begin{array}{l}\text { Understanding the agendas } \\
\text { and perspectives of owners, } \\
\text { staff members, managers, } \\
\text { and other parties }\end{array}$ & $\begin{array}{l}\text { Employing a team approach } \\
\text { to solve problems when } \\
\text { appropriate }\end{array}$ & Providing effective feedback \\
\hline $\begin{array}{l}\text { Considering customer needs } \\
\text { when making decisions }\end{array}$ & $\begin{array}{l}\text { Listening carefully to input } \\
\text { and concerns expressed by } \\
\text { others }\end{array}$ & $\begin{array}{l}\text { Demonstrating empathy in } \\
\text { dealing with customers and } \\
\text { staff }\end{array}$ \\
\hline $\begin{array}{l}\text { Bringing together different } \\
\text { perspectives and approaches } \\
\text { and combining them in } \\
\text { creative ways }\end{array}$ & $\begin{array}{l}\text { Promoting respect and } \\
\text { appreciation for diversity } \\
\text { and individual differences }\end{array}$ & $\begin{array}{l}\text { Manifesting intercultural } \\
\text { sensitivity }\end{array}$ \\
\hline
\end{tabular}

Source: Authors' research

\section{CONCLUSION}

The work presented demonstrates the burgeoning of the scholarly literature related to soft skills in $\mathrm{T} \& \mathrm{H}$, reflecting the importance of these skills for the sector. Moreover, it revealed the extensiveness and complexity of the potential soft skills list. Few previous scholarly works have conducted adequate methodological procedures to extract the most important soft skills for T\&H graduates (Chung-Herrera et al. 2003; Weber et al. 2013). However, the volume of literature in the after period requires a comprehensive and thorough SLR for defining the most critical soft skills required in the current T\&H market.

The resulting top 30 soft skill list derived by the SLR based on the CDM is the key theoretical contribution of this study. Furthermore, the study has significant practical implications for the T\&H industry and T\&H HEIs which are simultaneously the recommendations for future research on the subject. Namely, the resulting lists need to 
ToSEE - Tourism in Southern and Eastern Europe, Vol. 6, pp. 637-656, 2021.

Lj. Pranić, S. Pivčević, D. Garbin Praničević: TOP 30 SOFT SKILLS IN TOURISM AND ...

be empirically validated, i.e. used to evaluate the importance of the key soft skills and to what extent $\mathrm{T} \& \mathrm{H}$ students and graduates possess them after their studies completion. Various relevant stakeholders (academia, T\&H business sector and graduates) need to be included in the empirical studies to detect "skill gaps" that the T\&H study programmes should bridge.

Namely, it is widely accepted that collaboration among all T\&H stakeholders "is required to enable the development of a common understanding of the expectations and outcomes of tourism education" (Anderson 2018, 13). As soon as T\&H faculty, administrators, consultants, and industry practitioners capture and understand the key soft skills, the potential gap between the requirements of the $\mathrm{T} \& \mathrm{H}$ industry and tourism curricula adopted by HEIs can begin to be bridged.

Furthermore, studies in diverse $\mathrm{T} \& \mathrm{H}$ businesses and different countries/contexts are recommended to enable the much-needed comparisons and benchmarking. In the meantime, in line with calls from other studies on the need for the acquisition of crosscurricular or interdisciplinary skills (Kim et al. 2017), T\&H HEIs need to advance the attainment of soft skills in their curricula and classrooms. Furthermore, HEIs should connect tighter with $\mathrm{T} \& \mathrm{H}$ business and create opportunities for students to develop their soft skills outside of the classrooms.

\section{Limitations of the study}

This study has several potential limitations. First is the use of Goole Scholar. While it significantly outperforms other well-known academic search engines (i.e., Dimensions, EbscoHost, Microsoft Academic, OpenCitation's COCI, Proquest, Scopus, Web of Science, etc.) in terms of coverage (Gusenbauer, 2019; Martin-Martin et al., 2021, 2018a, $2018 \mathrm{~b}$ ), it is not flawless. Notably, it has a 256 character limit (with spaces) for searches, it does not provide the criteria for what makes its results "scholarly," and its search algorithm is not known and cannot be controlled. Moreover, since Google Scholar adapts the search to each user to personalize information, an SLR is difficult to replicate. However, through an in-depth analysis of Google Scholar's search results, especially when aided by multiple researchers, as in the case of this study, its benefits significantly outweigh the drawbacks. The second limitation is the key soft skills selection based on the views of HEI instructors. Although all researchers involved in the process have more than 15 years of experience in the HEI and T\&H research each, still their view of the key soft skills may differ from the views of $\mathrm{T} \& \mathrm{H}$ practitioners and students. The future studies should address this limitation.

\section{ACKNOWLEDGEMENTS}

This paper is partially based on research conducted within the INCOME Tourism Project, No. 588476-EPP-1-2017-1-PT-EPPKA2-KA, funded within ERAMSUS + programme of the European Commission. The authors acknowledge all the project consortium partners who contributed to the research. The study reflects the opinions and attitudes of the authors exclusively, and not those of the project partners nor those of the ERASMUS + Program. 
ToSEE - Tourism in Southern and Eastern Europe, Vol. 6, pp. 637-656, 2021.

Lj. Pranić, S. Pivčević, D. Garbin Praničević: TOP 30 SOFT SKILLS IN TOURISM AND ...

\section{REFERENCES}

Adeyinka-Ojo, S. (2018), “A strategic framework for analysing employability skills deficits in rural hospitality and tourism destinations", Tourism Management Perspectives, Vol. 27, pp. 47-54 https://doi.org/10.1016/j.tmp.2018.04.005

Anderson, W. and Sanga, J.J. (2019), “Academia-industry partnerships for hospitality and tourism education in Tanzania", Journal of Hospitality \& Tourism Education, Vol. 31, No. 1, pp. 34-48. https://doi.org/10.1080/10963758.2018.1480959

Andrades, L. and Dimanche, F. (2019), “Destination competitiveness in Russia: tourism professionals' skills and competences", International Journal of Contemporary Hospitality Management, Vol. 31, No. 2, pp. 910-930. https://doi.org/10.1108/IJCHM-11-2017-0769

Bach, S.A. and Milman, A. (1996)," A novel technique for reviewing a hospitality management curriculum", Hospitality \& Tourism Educator, Vol. 8, No. 1, pp. 37-40. https://doi.org/10.1080/23298758.1996.10685709

Baum, T. (1990), "Competencies for hotel management: Industry expectations of education”, International Journal of Contemporary Hospitality Management, Vol. 2, No. 4. https://doi.org/10.1108/09596119010135190

Baum, T. (2007), "Skills and the Hospitality Sector in a Transition Economy: The case of front office employment in Kyrgyzstan. Asia Pacific Journal of Tourism Research”, Vol. 12, No. 2, pp. 89-102. https://doi.org/10.1080/10941660701243315

Baum, T. and Thompson, K. (2007), "Skills and labour markets in transition: A tourism skills inventory of Kyrgyzstan, Mongolia and Uzbekistan”, Asia Pacific Journal of Human Resources, Vol. 45, No. 2 pp. 235-255. doi: 10.1177/1038411107073601

Bello, F.G., Kamanga, G. and Jamu, E.S. (2019), "Skills gaps and training needs in the tourism sector in Malawi”, African Journal of Hospitality, Tourism and Leisure, Vol. 8, No. 4, pp. 1-18.

Bharwani, S. and Jauhari, V. (2013), "An exploratory study of competencies required to co-create memorable customer experiences in the hospitality industry", International Journal of Contemporary Hospitality Management, Vol. 25 No. 6, pp. 823-843. https://doi.org/10.1108/IJCHM-05-2012 0065

Bharwani, S. and Talib, P. (2017), "Competencies of hotel general managers: a conceptual framework", International Journal of Contemporary Hospitality Management, Vol. 29 No. 1, pp. 393 418. https://doi.org/10.1108/IJCHM-09-2015-0448

Brownell, J. (1994), "Women in hospitality management: General managers' perceptions of factors related to career development", International Journal of Hospitality Management, Vol. 13, No. 2, pp. 101117. https://doi.org/10.1016/0278-4319(94)90032-9

Brownell, J. (2008), "Leading on land and sea: Competencies and context", International Journal of Hospitality Management, Vol. 27, No. 2, pp. 137-150. doi: 10.1016/j.ijhm.2007.11.003

Burns, P.M. (1997), "Hard-skills, soft-skills: undervaluing hospitality's 'service with a smile", Progress in Tourism and Hospitality Research, Vol. 3, No. 3, pp. 239-248. https://doi.org/10.1002/(SICI)10991603(199709)3:3<239::AID-PTH80>3.0.CO;2-2

Bustreo, M., Micheletto, V., Quaratino, L. and Fiorentino, D. (2018)," The impact of successful crosscompetencies on a career in tourism in Italy: the meeting point between the students' perceptions and the requirements for professionals", Journal of Teaching in Travel \& Tourism, Vol 18, No.3, pp.179-201. https://doi.org/10.1080/15313220.2018.1460652

Caruana, S. and Mcpherson, M. (2015), "A constructive alignment approach for assessing essential cultural soft skills in tourism”, Procedia-Social and Behavioral Sciences, Vol. 191, pp. 6-11. doi: 10.1016/j.sbspro.2015.04.404

Cassidy, S. (2006), "Developing employability skills: Peer assessment in higher education", Education + Training, Vol. 48, No. 7, pp. 508-517. https://doi.org/10.1108/00400910610705890

Causin, G.F. and Ayoun, B. (2011), "Packing for the trip: A model of competencies for successful expatriate hospitality assignment", International Journal of Hospitality Management, Vol.30, No.4, pp.795802. doi: 10.1016/j.ijhm.2010.12.011

Chen, T.L., Shen, C.C. and Gosling, M. (2021), "To stay or not to stay? The causal effect of interns' career intention on enhanced employability and retention in the hospitality and tourism industry", Journal of Hospitality Leisure, Sport \& Tourism Education, Vol. 28, 100305. https://doi.org/10.1016/j.jhlste.2021.100305

Cheung, C., Law, R. and He, K. (2010), "Essential hotel managerial competencies for graduate students", Journal of Hospitality \& Tourism Education, Vol. 22, No. 4, pp. 25-32. https://doi.org/10.1080/10963758.2010.10696989

Christou, E. and Chatzigeorgiou, C. (2019), "Industrial placement in hospitality management education. Students' experiences and development of skills", in ICOMEU conference proceedings, 2nd 
ToSEE - Tourism in Southern and Eastern Europe, Vol. 6, pp. 637-656, 2021.

Lj. Pranić, S. Pivčević, D. Garbin Praničević: TOP 30 SOFT SKILLS IN TOURISM AND ..

international congress on management of educational units, 29th November-1st December 2019 Thessaloniki, Greece, pp.157-164. http://dx.doi.org/10.5281/zenodo.3755091

Chung-Herrera, B.G., Enz, C.A. and Lankau, M.J. (2003), "Grooming future hospitality leaders: A competencies model”, Cornell Hotel and Restaurant Administration Quarterly, Vol. 44, No. 3, pp. 17-25. https://doi.org/10.1016/S0010-8804(03)90266-7

Cimatti, B. (2016), "Definition, development, assessment of soft skills and their role for the quality of organizations and enterprises", International Journal for Quality Research, Vol. 10, No. 1, pp. 97 130. doi:10.18421/IJQR10.01-05

Clark, M. (1993), "Communications and social skills: Perceptions of hospitality managers", Employee Relations, Vol. 15, No. 2, pp. 51-60. https://doi.org/10.1108/0142545931003182

Costa, V., Moura, A., Mira, M.D.R., Cerdeiras, A., Cruz, I.P.D., Pereira, J.F., Martins, I.S., Conceição, O., Vilaça, A., Almeida, C. and Carrança, P. (2019), "HC Tourism. Profile and trends of human capital in the tourism sector", European Journal of Tourism, Hospitality and Recreation, Vol. 9, No. 2, pp. 40-51. https://doi.org/10.2478/ejthr-2019-0009

Davies, J. (2000), “A study of language skills in the leisure and tourism industry”. Language Learning Journal, Vol. 21. No. 1, pp. 66-71. http://dx.doi.org/10.1080/09571730085200131

DeVellis, R.F. (2003), Scale Development: Theory and Applications, Sage Publications, Thousand Oaks, CA DeVellis, R.F. (1991), Scale Development: Theory and Applications, Sage Publications, Newbury Park, CA.

Deale, C.S. and Lee, S.H. (2021), “An exploratory study of hospitality and tourism stakeholders' perceptions of professional etiquette”, Journal of Hospitality \& Tourism Education, Vol. 33, No. 1, pp. 43-56. doi: 10.1080/10963758.2019.1654891

Denizci Guillet, B., Pavesi, A., Hsu, C. and Weber, K. (2019), "What can educators do to better prepare women for leadership positions in the hospitality industry?, The perspectives of women executives in Hong Kong", Journal of Hospitality \& Tourism Education, Vol. 31, No. 4, pp. 197 209. https://doi.org/10.1080/10963758.2019.1575751

Dhiman, M.C. (2012), "Employers' perceptions about tourism management employability skills", Anatolia Vol. 23, No. 3, pp. 359-372. http://dx.doi.org/10.1080/13032917.2012.711249

Fraser, B. (2020), "From Hospitality Classrooms to Successful Careers: A Current Appraisal of Australian International Hotel Requirements", Journal of Hospitality and Tourism Education, Vol. 32. No. 4, pp. 234-254. https://doi.org/10.1080/10963758.2019.1688161

Ferreras-Garcia, R., Sales-Zaguirre, J., and Serradell-López, E. (2019), "Competences in higher education tourism internships”, Education and Training, Vol. 62, No. 1, pp. 64-80 https://doi.org/10.1108/ET-04-20190074

Foster, C., McCabe, S. and Dewhurst, H. (2010), "Management development skills in the hospitality and tourism sector: Needs and issues from a regional perspective", Tourism and Hospitality Planning \& Development, Vol. 7, No. 4, pp. 429-445. https://doi.org/10.1080/1479053X.2010.523176

Ginaya, G., Kanca, I.N., and Sri Astuti, N.N. (2020), "Designing problem-based learning (PBL) model for tourism vocational education in 4.0 industry", International Journal of Linguistics, Literature and Culture, Vol. 6, No. 1, pp. 14-23. https://doi.org/10.21744/ijllc.v6n1.808

Gusenbauer, M. (2019), "Google Scholar to overshadow them all? Comparing the sizes of 12 academic search engines and bibliographic databases", Scientometrics, Vol. 118, pp. 177-214. https://doi.org/10.1007/s1119 2-018-2958-5

Hannam, K., Mitsche, N. and Stone, C. (2004), "Tourism employability and the European social fund", in Proceedings of the 2004 Conference Critical Issues in Tourism Education, Association for Tourism in Higher Education, pp. 39-52.

Hassan, S.N.S., Kamarudin, M.F., Rajikon, M.A.N., Saadan, R., Yunus, A.R., Mustapha, R. and Omar, M. (2009), "The importance of soft skills in tourism industry in Melaka Malaysia", Journal of Human Capital Development, Vol. 2, No. 2, pp. 37-48.

Hertzman, J.L., Moreo, A.P. and Wiener, P.J. (2015), "Career planning strategies and skills of hospitality management students", Journal of Human Resources in Hospitality \& Tourism, Vol. 14, No. 4, pp. 423-443 http://dx.doi.org/10.1080/15332845.2015.1002071

Horng, J. and Lu, H. (2006), "Needs assessment of professional competencies of F\&B / hospitality management students at college and university level", Journal of Teaching in Travel and Tourism, Vol. 6, No. 3 , pp. 1-26. http://dx.doi.org/10.1300/J172v06n03_01

Hossny, M. F. (2018), "A Gap Analysis of the Essential Competencies Expected and Perceived from Hospitality Graduates in the Industry : The Case of Egypt", International Academic Journal of Faculty of Tourism and Hospitality Management, Vol. 4, No. 4, pp. 93-115. https://doi.org/10.21608/IJAF.2018.95501 
ToSEE - Tourism in Southern and Eastern Europe, Vol. 6, pp. 637-656, 2021.

Lj. Pranić, S. Pivčević, D. Garbin Praničević: TOP 30 SOFT SKILLS IN TOURISM AND ...

Huang, Y., Lalopa, J.M. and Adler, H. (2016), "An analysis of entry level management requirements: Are there differences in perceptions of hospitality recruiters versus hospitality students?", Journal of Human Resources in Hospitality \& Tourism, Vol. 15, No. 3, pp. 346-364. http://dx.doi.org/10.1080/15332845.2016.1147980

Huang, Y-L. and Lin, C-T. (2010), "Management trainee core competencies in the hospitality industry: differences between managers and scholars", Journal of Human Resources in Hospitality and Tourism, Vol. 10 No. 1, pp. 1-13. https://doi.org/10.1080/15332845.2010.500166

Jack, K., Stansbie, P. and Sciarini, M. (2017), "An examination of the role played by internships in nurturing management competencies in Hospitality and Tourism Management (HTM) students", Journal of Teaching in Travel \& Tourism, Vol. 17, No. 1, pp. 17-33. http://dx.doi.org/10.1080/15313220.2016.1268946

Jiang, L. and Alexakis, G. (2017), "Comparing students' and managers' perceptions of essential entry-level management competencies in the hospitality industry: An empirical study", Journal of Hospitality, Leisure, Sport \& Tourism Education, Vol. 20, pp. 32-46. http://dx.doi.org/10.1016/j.jhlste.2017.01.001

Kalargyrou, V. and Woods, R.H. (2011), "Wanted: Training competencies for the twenty-first century", International Journal of Contemporary Hospitality Management, Vol. 23, No. 3, pp. 361-376. http://dx.doi.org/10.1108/09596111111122532

Kay, C. and Russette, J. (2000), “Hospitality management competencies: Identifying managers' essential skills", Cornell Hotel and Restaurant Administration Quarterly, Vol. 41, No. 2, pp. 52-63. doi:10.1016/S0010-8804(00)88898-9

Khan, K.S., Kunz, R., Kleijnen, J. and Antes, G. (2003), "Five steps to conducting a systematic review", Journal of the Royal Society of Medicine, Vol. 96, pp. 118-121.

Kim, N., Park, J., and Choi, J.J. (2017), "Perceptual differences in core competencies between tourism industry practitioners and students using Analytic Hierarchy Process (AHP)". Journal of Hospitality, Leisure, Sport and Tourism Education, Vol. 20, pp. 76-86. https://doi.org/10.1016/j.jhlste.2017.04.003.

Kimeto, J.C. (2021), "Tertiary tourism graduate employees and tourism employers' perceptions on tourism skills and competencies relevant for providing quality tourism services in Kenya", Tourism Critiques: Practice and Theory, Vol. 2, No. 1, pp. 20-37. https://doi.org/10.1108/TRC-07-20200013

Ko, W. (2010), "To Evaluate the Professional Culinary Competence of Hospitality Students", Journal of Culinary Science \& Technology, Vol. 8, pp. 136-146. https://doi.org/10.1080/15428052.2010.511101

Kriegl, U. (2000), "International hospitality management: Identifying important skills and effective training", Cornell Hospitality Quarterly, Vol. 41, No. 2, pp. 64-71. https://doi.org/10.1177/001088040004100218

Kumar, S.V. (2021), “A Study on Adept Soft Skill \& Knowledge Accompanying Hospitality Employee Retention Behavior During Covid-19 Lockdown Phase", Anais Brasileiros de Estudos Turísticos ABET.

Lin, S-C. (2002), "Exploring the relationships between hotel management courses and industry required competencies", Journal of Teaching in Travel \& Tourism, Vol. 2, No. 3/4, pp. 81-101. https://doi.org/10.1300/J172v02n03_05

Lolli, J.C. (2013), "Interpersonal communication skills and the young hospitality leader: Are they prepared?", International Journal of Hospitality Management, Vol. 32, No. 1, pp. 295-298. http://dx.doi.org/10.1016/j.ijhm.2012.02.010

Luka, I. (2015), "Enhancing employability skills for tourism and hospitality industry employees in Europe", Acta Prosperitatis, Vol. 6, pp. 75-94.

Marneros, S., Papageorgiou, G. and Efstathiades, A. (2020), "Identifying key success competencies for the hospitality industry : the perspectives of professionals", Journal of Teaching in Travel \& Tourism, Vol. 20, No. 4, pp. 237-261. https://doi.org/10.1080/15313220.2020.1745732

Marneros, S., Papageorgiou, G. and Efstathiades, A. (2021), "Sport \& Tourism Education Examining the core competencies for success in the hotel industry: The case of Cyprus", Journal of Hospitality, Leisure, Sport \& Tourism Education, Vol. 28, 100303. https://doi.org/10.1016/j.jhlste.2021.100303

Martín-Martín, A., Thelwall, M., Orduna-Malea, E. and Delgado López-Cózar, E. (2021), "Google Scholar, Microsoft Academic, Scopus, Dimensions, Web of Science, and OpenCitations' COCI: A multidisciplinary comparison of coverage via citations", Scientometrics, Vol. 126, pp. 871-906. https://doi.org/10.1007/s11192-020-03690-4

Martín-Martín, A., Orduna-Malea, E. and Delgado López-Cózar, E. (2018a), "Coverage of highly-cited documents in Google Scholar, Web of Science, and Scopus: A multidisciplinary comparison", Scientometrics, Vol. 116, pp. 2175-2188. https://doi.org/10.1007/s11192-018-2820-9 
ToSEE - Tourism in Southern and Eastern Europe, Vol. 6, pp. 637-656, 2021.

Lj. Pranić, S. Pivčević, D. Garbin Praničević: TOP 30 SOFT SKILLS IN TOURISM AND

Martín-Martín, A., Orduna-Malea, E., Thelwall, M. and Delgado López-Cózar, E. (2018b), "Google Scholar, Web of Science, and Scopus: A systematic comparison of citations in 252 subject categories", Journal of Informetrics, Vol. 12, No. 4, pp. 1160-1177. https ://doi.org/10.1016/J.JOI.2018.09.002

Millar, M., Mao, Z. and Moreo, P. (2010), "Hospitality \& tourism educators vs. the industry: A competency assessment", Journal of Hospitality \& Tourism Education, Vol. 22, No. 2, pp. 38-50 http://dx.doi.org/10.1080/10963758.2010.10696975

Moolman, H.J. and Wilkinson, A. (2014), "Essential generic attributes for enhancing the employability of hospitality management graduates", Tourism, Vol. 62, No. 3, pp. 257-276.

Munar, A.M. and Montaño, J.J. (2009), "Generic competences and tourism graduates", Journal of Hospitality, Leisure, Sport and Tourism Education, Vol. 8., No. 1, pp. 70-84. doi:10.3794/johlste.81.206

Nickson, D., Warhurst, C. and Dutton, E. (2005), „The importance of attitude and appearance in the service encounter in retail and hospitality“, Managing Service Quality: An International Journal. Vol. 15, No. 2, pp. 195 - 208. https://doi.org/10.1108/09604520510585370

Raybould, M. and Wilkins, H. (2006), "Generic skills for hospitality management: A comparative study of management expectations and student perceptions", Journal of Hospitality and Tourism Management, Vol. 13, No. 2, pp. 177-188. https://doi.org/10.1375/jhtm.13.2.177

Robinson, J.S. and Garton, B.L. (2008), "An assessment of the employability skills needed by graduates in the college of agriculture, food and natural resources at the University of Missouri", Journal of Agricultural Education, Vol. 49, No. 4, pp. 96-105.

Robles, M.M. (2012), "Executive perceptions of the top 10 soft skills needed in today's workplace", Busines Communication Quarterly, Vol. 75, No. 4, pp. 453-465. https://doi.org/10.1177/1080569912460400

Patiar, A., Kensbock, S., Benckendorff, P., Robinson, R., Richardson, S., Wang, Y. and Lee, A. (2021), "Hospitality students' acquisition of knowledge and skills through a virtual field trip experience", Journal of Hospitality \& Tourism Education, Vol. 33, No. 1, pp. 14-28. https://doi.org/10.1080/10963758.2020.1726768

Petkovski, K. (2012), „Required skills and leadership characteristics of a modern manager in tourism and hospitality“, UTMS Journal of Economics, Vol. 3, No. 1, pp .91-96.

Sandwith, P. (1993), “A hierarchy of management training requirements: The competency domain model”, Public Personnel Management, Vol. 22, No. 1, pp. 43-62. https://doi.org/10.1177/009102609302200104

Scarinci, J. and Pearce, P. (2012), ,The perceived influence of travel experiences on learning generic skills“, Tourism Management, Vol. 33, No. 2, pp. 380-386. https://doi.org/10.1016/j.tourman.2011.04.007

Sharma, S. and Sharma, R. (2019), "Culinary skills: the spine of the Indian hospitality industry: Is the available labor being skilled appropriately to be employable?", Worldwide Hospitality and Tourism Themes, Vol. 11, No. 1, pp. 25-36. https://doi.org/10.1108/WHATT-10-2018-0061

Shay, J.P. and Tracey, J.B. (1997), "Expatriate managers: Reasons for failure and implications for training", Cornell Hotel and Restaurant Administration Quarterly, Vol. 38, No. 1, pp. 30-35 doi:10.1016/S0010-8804(97)87489-7

Shereni, N.C. (2020), "The role of technical and vocational education and training (TVET) in restoring hospitality sector specific skills in Zimbabwe: a students' perspective", Journal of Hospitality \& Tourism Education, Vol. 32, No. 3, pp. 133-141. https://doi.org/10.1080/10963758.2019.1655434

Shum, C., Gatling, A. and Shoemaker, S. (2018), „A model of hospitality leadership competency for frontline and director-level managers: Which competencies matter more?“, International Journal of Hospitality Management, Vol. 74, pp. 57-66. https://doi.org/10.1016/j.ijhm.2018.03.002

Sisson, L.G. and Adams, A.R. (2013), "Essential hospitality management competencies: The importance of soft skills", Journal of Hospitality \& Tourism Education, Vol. 25, No. 3, pp. 131-145. https://doi.org/10.1080/10963758.2013.826975

Siu, V. (1998), "Managing by competencies - a study on the managerial competencies of hotel middle managers in Hong Kong", Hospitality Management, Vol. 17, pp. 253-273. https://doi.org/10.1016/S0278-4319(98)00041-3

Spowart, J. (2011), "Hospitality students' competencies: Are they work ready?", Journal of Human Resources in Hospitality \& Tourism, Vol. 10, No. 2, pp. 169-181. https://doi.org/10.1080/15332845.2011.536940

Succi, C. 2019, "Are you ready to find a job? Ranking of a list of soft skills to enhance graduates' employability", International Journal of Human Resources Management and Development, Vol. 19, No. 3, pp. 281-297.

Succi, C. and Canovi, M. (2020), "Soft skills to enhance graduate employability: Comparing students and employers" perceptions", Studies in Higher Education, Vol. 45, No. 9, pp. 1834-1847. https://doi.org/10.1080/03075079.2019.1585420

Suh, E., West, J.J. and Shin, J. (2012), „Important competency requirements for managers in the hospitality industry“, Journal of hospitality, leisure, sport \& tourism education, Vol. 11, No. 2, pp. 101-112. https://doi.org/10.1016/j.jhlste.2012.02.005 
ToSEE - Tourism in Southern and Eastern Europe, Vol. 6, pp. 637-656, 2021.

Lj. Pranić, S. Pivčević, D. Garbin Praničević: TOP 30 SOFT SKILLS IN TOURISM AND ...

Susaeta, L., Babinger, F. and Muñoz, N. (2020)," Influence of limiting beliefs in soft employability skills: An analysis for the hospitality sector", Tourism: An International Interdisciplinary Journal, Vol. 68, No. 2, pp. 207-220.

Tas, R.F., LaBrecque, S.V. and Clayton, H.R. (1996), "Property-management competencies for managemen trainees", Cornell Hotel and Restaurant Administration Quarterly, Vol. 37, No. 4, pp. 90-96. https://doi.org/10.1177/001088049603700427

Temİzkan, R. (2020), "Towards Qualified Human Resources in the Hospitality Industry : A Review of the Hospitality Management Competencies", Ege Academic Review, Vol. 20, No. 3, pp. 153-165. https://doi.org/10.21121/eab

Teng, W., Ma, C., Pahlevansharif, S., and Turner, J. J. (2019), "Graduate readiness for the employment market of the 4th industrial revolution: The development of soft employability skills", Education and Training, Vol. 61, No. 5, pp. 590-604. https://doi.org/10.1108/ET-07-2018-0154.

Tesone, D.V., and Ricci, P. (2006), "Toward a Definition of Entry-Level Job Competencies", International Journal of Hospitality \& Tourism Administration, Vol. 7, No. 4, pp. 65-80. https://doi.org/10.1300/j149v07n04 04

Tsai, F.C., Goh, B.K., Huffman, L. and Wu, K. (2006), “Competency assessment for entry-level lodging management trainees in Taiwan", The Chinese Economy, Vol. 39, No. 6, pp. 49-69. https://doi.org/10.2753/CES1097-1475390604

Valdez, E.J.C., Alcantara, S.S.B., Pamintuan, C.A., Relos, J.G., and Castillo, R.C. (2015), “Contributions of On-the-Job Training Program to the Skills, Personal Qualities and Competencies of Tourism Graduates", Asia Pacific Journal of Multidisciplinary Research, Vol. 3, No. 4, pp. 102-110.

Vučetić, A.Š. (2018), "Differences in perception of the importance of generic competencies among destination regions", Economic Research-Ekonomska Istraživanja, Vol. 31, No. 1, pp. 1-18. https://doi.org/10.1080/1331677X.2018.1482224

Wang, Y.-F. (2013), "Constructing career competency model of hospitality industry employees for career success", International Journal of Contemporary Hospitality Management, Vol. 25, No. 7, pp. 994 1016. https://doi.org/10.1108/IJCHM-07-2012-0106

Wang, Y-F. and Tsai, C-T. (2014), "Employability of hospitality graduates: Student and industry perspectives", Journal of Hospitality \& Tourism Education, Vol. 26, No. 3, pp. 125-135.

Weber, M.R., Crawford, A., Lee, J. and Dennison, D. (2013), "An exploratory analysis of soft skil competencies needed for the hospitality industry", Journal of Human Resources in Hospitality \& Tourism, Vol. 12, No. 4, pp. 313-332. https://doi.org/10.1080/15332845.2013.790245

Weber, M.R., Finley, D.A., Crawford, A. and River, D.Jr. (2009), “An exploratory study identifying soft skill competencies in entry-level managers", Tourism and Hospitality Research, Vol. 9, No. 4, pp. 353 361. https://doi.org/10.1057/thr.2009.22

Wesley, S.C., Jackson, V.P., and Lee, M. (2017), "The perceived importance of core soft skills between retailing and tourism management students, faculty and businesses", Employee Relations, Vol. 39 No. 1, pp. 79-99. https://doi.org/10.1108/ER-03-2016-0051

Wilks, D. and Hemsworth, K. (2011), "Soft skills as key competencies in hospitality higher education: Matching demand and supply", Tourism \& Management Studies, Vol. 7, pp. 131-139.

WTTC (2020) Benchmarking Research Trends 2019.

Yang, H., Cheung, C. and Fang, C.C. (2015), “An Empirical Study of Hospitality Employability Skills: Perceptions of Entry-Level Hotel Staff in China", Journal of Hospitality and Tourism Education, Vol. 27, No. 4, pp. 161-170. https://doi.org/10.1080/10963758.2015.1089510

Yang, H., Cheung, C. and Song, H. (2016), “Enhancing the learning and employability of hospitality graduates in China”, Journal of Hospitality, Leisure, Sport and Tourism Education, Vol. 19, pp. 85-96. https://doi.org/10.1016/j.jhlste.2016.08.004

Yang, L.-T. (Grace), Partlow, C.G., Anand, J., and Shukla, V. (2014), “Assessing the Competencies Needed by Hospitality Management Graduates in India", Journal of Hospitality \& Tourism Education, Vol 26, No. 4, pp. 153-165. https://doi.org/10.1080/10963758.2014.959963

Yorke, M. (2006), Employability in Higher Education: What it is - What it is Not, The Higher Education Academy, viewed 26 March 2021, https://www.ed.ac.uk/files/atoms/files/hea-learning employability_series_one.pdf

Zehrer, A. and Mössenlechner, C. (2009), "Key competencies of tourism graduates: The employers' point of view", Journal of Teaching in Travel \& Tourism, Vol. 9, No. 3/4, pp. 266-287. https://doi.org/10.1080/15313220903445215 
ToSEE - Tourism in Southern and Eastern Europe, Vol. 6, pp. 637-656, 2021.

Lj. Pranić, S. Pivčević, D. Garbin Praničević: TOP 30 SOFT SKILLS IN TOURISM AND ..

Ljudevit Pranić, PhD, Associate Professor

University of Split, Faculty of Economics, Business and Tourism

Department of Tourism and Economy

Cvite Fiskovića 5, 21000 Split, Croatia

+385-21-430749

ljudevit.pranic@efst.hr

Smiljana Pivčević, PhD, Associate Professor

University of Split, Faculty of Economics, Business and Tourism

Department of Tourism and Economy

Cvite Fiskovića 5, 21000 Split, Croatia

+385-21-430669

smipi@efst.hr

Daniela Garbin Praničević, PhD, Associate Professor

University of Split, Faculty of Economics, Business and Tourism

Department of Business Informatics

Cvite Fiskovića 5, 21000 Split, Croatia

+385-21-430612

daniela@efst.hr 